\title{
Quantization of Gravitational Wave by Klein-Gordon Equation
}

\author{
Sangwha-Yi* \\ Department of Math, Taejon University 300-716, South Korea
}

*Corresponding Author: Sangwha-Yi, Department of Math, Taejon University 300-716, South Korea

Abstract: In the general relativity theory, we find gravitational matter wave by Klein-Gordon wave equation. Specially, this article is that Quantization of gravitational wave is made by Klein-Gordon wave equation. We assume this matter wave as Dark Matter.

Keywords: General relativity theory, Gravitational Wave; Klein-Gordon wave equation; Gravitational matter wave; Dark matter

PACS Number: 04,04.90.+e,03.30, 41.20

\section{INTRODUCTION}

In the general relativity theory, our article's aim is that we find the quantization of gravitational wave by Klein-Gordon wave equation.

At first, gravitational wave equation is

$$
\left.\nabla^{2}-\frac{1}{c^{2}} \frac{\partial^{2}}{\partial t^{2}}\right) h_{\mu v}=0
$$

The solution, gravitational wave function $h_{\mu v}(x)$ is

$$
h_{\mu v}(x)=a_{\mu v} \exp \left(k_{\lambda} x^{\lambda}\right)+a_{\mu \nu}^{*} \exp \left(-i k_{\lambda} x^{\lambda}\right)
$$

In this time,

$k_{\lambda} k^{\lambda}=-\frac{\omega_{0}^{2}}{c^{2}}+k_{0}^{2}=0, k_{\lambda}=\left(\frac{\omega_{0}}{c}, \vec{k}_{0}\right), k^{\lambda}=\left(-\frac{\omega_{0}}{c}, \vec{k}_{0}\right)$

The constant tensor $a_{\mu v}$ is the polarization tensor.

$$
a_{\mu v}=a_{v \mu}
$$

Harmonic coordinate condition is

$$
\begin{aligned}
& \left(\nabla^{2}-\frac{1}{c^{2}} \frac{\partial^{2}}{\partial t^{2}}\right) \varepsilon_{v}=\frac{\partial h^{\mu}{ }_{v}}{\partial x^{\mu}}-\frac{1}{2} \frac{\partial h_{\mu}^{\mu}}{\partial x^{v}} \\
& k_{\mu} a_{\nu}^{\mu}=\frac{1}{2} k_{v} a_{\mu}^{\mu}
\end{aligned}
$$

The coordinate transformation is

$$
x^{\mu}=x^{\mu}+i \varepsilon^{\mu} \exp \left(k_{\lambda} x^{\lambda}\right)-i \varepsilon^{\star \mu} \exp \left(-i k_{\lambda} x^{\lambda}\right)
$$


According to $\operatorname{Eq}(7)$, the transformation of the polarization tensor is

$a_{\mu v}^{\prime}=a_{\mu v}+k_{\mu} \varepsilon_{v}+k_{v} \varepsilon_{\mu}$

\section{Quantization of GRaVitational WAVE by KLEIN-GoRdon EQuation}

The speed of Gravitational wave is light speed. If we make matter by Gravitational space-time, this matter moves as the usual matter. We consider the matter interacting only gravity. Hence, we assume this matter as Dark Matter.

At first, gravitational matter wave equation is

$$
\left.\nabla^{2}-\frac{1}{c^{2}} \frac{\partial^{2}}{\partial t^{2}}\right) h_{\mu v}=\frac{m_{0}{ }^{2} C^{2}}{\hbar^{2}} h_{\mu v}
$$

The solution, gravitational matter wave function $h_{\mu v}(x)$ is

$$
h_{\mu \nu}(x)=a_{\mu v} \exp \left(k_{\lambda} x^{\lambda}\right)+a_{\mu \nu}^{*} \exp \left(-k_{\lambda} x^{\lambda}\right)
$$

In this time,

$$
\begin{aligned}
& -k_{\lambda} k^{\lambda}=\frac{\omega_{0}^{2}}{c^{2}}-k_{0}^{2}=\frac{m_{0}^{2} c^{2}}{\hbar^{2}}, E=\hbar \omega_{0}, \vec{p}=\hbar \vec{k}_{0}, \\
& k_{\lambda}=\left(\frac{\omega_{0}}{C}, \vec{k}_{0}\right), k^{\lambda}=\left(-\frac{\omega_{0}}{c}, \vec{k}_{0}\right)
\end{aligned}
$$

The constant tensor $a_{\mu v}$ is the polarization tensor.

$$
a_{\mu v}=a_{v \mu}
$$

Harmonic coordinate condition is in gravitational matter wave by $\mathrm{Eq}(5), \mathrm{Eq}(6)$

$$
\left.k_{\mu} a^{\mu}{ }_{v}-\frac{1}{2} k_{v} a_{\mu}^{\mu}=\frac{m_{0}{ }^{2} C^{2}}{\hbar^{2}} \varepsilon_{v}=\nabla^{2}-\frac{1}{c^{2}} \frac{\partial^{2}}{\partial t^{2}}\right) \varepsilon_{v}
$$

\section{CONCLUSiON}

We find the gravitational matter wave by Klein-Gordon wave equation. We find the quantization of gravitational wave by Klein-Gordon wave equation.

\section{REFERENCES}

[1]S.Yi, "Electromagnetic Wave Functions of CMB and Schwarzschild Space-Time", International Journal of Advanced Research in Physical Science,6,3,(2019)

[2]S.Yi, "Klein-Gordon Equation and Wave Function for Free Particle in Rindler Space-Time", International Journal of Advanced Research in Physical Science, 7,9,(2020)

[3]S.Weinberg,Gravitation and Cosmology(John wiley \& Sons,Inc,1972)

[4]W.Rindler, Am.J.Phys.34.1174(1966)

[5]P.Bergman,Introduction to the Theory of Relativity(Dover Pub. Co.,Inc., New York,1976), Chapter V [6]C.Misner, K,Thorne and J. Wheeler, Gravitation(W.H.Freedman \& Co.,1973)

[7]S.Hawking and G. Ellis,The Large Scale Structure of Space-Time(Cam-bridge University Press,1973) 
[8]R.Adler,M.Bazin and M.Schiffer,Introduction to General Relativity(McGraw-Hill,Inc.,1965)

[9]A. Einstein, “ Zur Elektrodynamik bewegter K“orper”, Annalen der Physik. 17:891(1905)

[10]J.D. Bjorken \& S. D. Drell, Relativistic Quantum Field(McGraw- Hill Co., 1965)

[11]P.Bergman,Introduction to the Theory of Relativity(Dover Pub. Co.,Inc., New York,1976), Chapter V

[12]R.L.Liboff, Quantum Mechanics(Addison-Wesley Publishing Co., Inc., 1990)

Citation: Sangwha-Yi, (2021). Quantization of Gravitational Wave by Klein-Gordon Equation. International Journal of Advanced Research in Physical Science (IJARPS) 8(1), pp.17-19, 2020.

Copyright: (C) 2021 Authors, This is an open-access article distributed under the terms of the Creative Commons Attribution License, which permits unrestricted use, distribution, and reproduction in any medium, provided the original author and source are credited. 